\title{
Robotic-assisted tubal anastomosis with one-stitch technique
}

\author{
Shahryar K. Kavoussi • K. M. Kavoussi • \\ Dan I. Lebovic
}

Received: 17 September 2013/ Accepted: 24 November 2013/Published online: 5 December 2013

(C) Springer-Verlag London 2013

\begin{abstract}
The da Vinci ${ }^{\circledR}$ robotic surgical system has been used more often in recent years for tubal anastomosis (TA) and has been reported to have an increased operative time. A one-stitch technique has been used for the reanastomosis step in laparoscopic TA. To date, publications on robotically-assisted TA (RATA) describe an anastomotic step with multiple (usually four) sutures placed. This retrospective case series reports tubal patency data on patients who underwent RATA with the one-stitch technique; tubal patency was the outcome measure. Eighteen women (ages 27-39) underwent RATA with the one-stitch anastomotic technique in tertiary care medical centers between February 2009 and May 2012. Tubal patency was demonstrated in $16 / 17$ patients $(94.1 \%$ ), as evidenced by postoperative hysterosalpingogram (HSG) and/or subsequent pregnancies. We report the first case series which shows that RATA with a single stitch for the reanastomotic step is effective in achieving tubal patency as evidenced by postoperative HSG and/or pregnancies.
\end{abstract}

S. K. Kavoussi $(\bowtie) \cdot$ K. M. Kavoussi

Austin Fertility and Reproductive Medicine/Westlake IVF, 300

Beardsley Lane, Bldg B, Suite 200, Austin, TX 78746, USA

e-mail: skavoussi@msn.com

\section{I. Lebovic}

Division of Reproductive Endocrinology and Infertility, Department of Obstetrics and Gynecology, University of Wisconsin, Madison, USA

D. I. Lebovic

Generations Fertility Care, 2365 Deming Way, Middleton, WI 53562, USA
Keywords Tubal ligation reversal - Tubal reanastamosis $\cdot$ Robotic-assisted $\cdot$ da Vinci robot . One-stitch

\section{Introduction}

Women who have a history of tubal ligation and desire a return to fertility have the options of tubal anastomosis (TA) or in vitro fertilization (IVF). TA has been found to be cost-effective for all women of reproductive age including those over 40 years of age [1]. For appropriate candidates who have proceeded with TA, the surgical approach to this procedure has evolved from an open laparotomy to laparoscopic TA and, most recently, robotically-assisted TA (RATA). The potential advantages of RATA over open laparotomy include the avoidance of a larger incision, shorter hospitalization time, and speedier postoperative recovery for patients, with comparable patency and pregnancy rates [2]. Moreover, the potential advantages of RATA over laparoscopic TA include shorter operative time, less technical difficulty due to enhanced ergonomics, and the minimization or elimination of surgeon fatigue when performing reversal on the second fallopian tube.

Several studies have examined the efficacy of RATA [2-7]. These studies have described the anastomosis step as using multiple (usually four) interrupted sutures to reapproximate the mucosal and muscular layers of the proximal and distal fallopian tube segments [2]. A one-stitch technique for the anastomosis step has been used effectively in laparoscopic TA [8, 9]. Unlike the aforementioned reports, this study goes further by combining the robotic approach along with the one-stitch technique for tubal anastomosis. Tubal patency, as evidenced by hysterosalpingogram 
(HSG) and/or subsequent pregnancy, was the primary outcome measure of interest, with operative time a secondary measure.

\section{Materials and methods}

Eighteen parous women with a history of tubal ligation who had opted for tubal anastomosis over IVF underwent da Vinci ${ }^{\circledR}$ robotically-assisted TA between February 2009 and May 2012. IRB approval was obtained from the Austin Multi-Institutional Review Board. The study outcome measure was tubal patency as demonstrated by hysterosalpingogram (HSG) and/or subsequent pregnancy.

The positioning of the patient and the robot were similar to methods described by Dharia Patel et al. [2]. Briefly, after general anesthesia was induced, the patient was placed in modified dorsal lithotomy position in steep Trendelenberg, with a Humi ${ }^{\mathrm{TM}}{ }^{\circledR}$ Harris-Kronner uterine manipulator-injector (CooperSurgical, Trumbull, CT, USA) for uterine mobilization and chromotubation. Peritoneal access was achieved with a $12-\mathrm{mm}$ umbilical trocar. Two lateral 8-mm da Vinci ports (Intuitive Surgical, Sunnyvale, CA, USA) were placed in the midaxillary line at the level of the umbilical port, separted by at least $8 \mathrm{~cm}$ between port sites. After diagnostic laparoscopy assessed the feasibility of anastomosis, a $10-\mathrm{mm}$ (8-mm for more recent cases) accessory port was placed between the umbilical port and left-sided da Vinci port for irrigation and aspiration as well as for the introduction and removal of suture material. The robot was then positioned and the robotic arms were docked to the ports.

The one-stitch technique of Dubuisson and Swolin, with the modifications described by Bissonnette, was used [8, 9]. Dilute vasopressin (1:60, Pitressin; Fujisawa, Ontario, Canada) was injected into the mesosalpinges in order to facilitate hydrodissection and to maximize hemostasis. The stumps of the occluded proximal fallopian segments were sectioned sharply with EndoWrist ${ }^{\circledR}$ Monopolar Curved Scissors until the lumen was visualized and indigo carmine injection revealed proximal patency. The occluded ends of the distal fallopian tubes were sectioned sharply with EndoWrist Monopolar Curved Scissors and exposure of the distal segments to indigo carmine that had been injected through the proximal segments facilitated the identification of the distal lumen by visualizing the uptake of indigo carmine into the distal lumen. Suture materials were then advanced into the surgical field through the accessory port. In order to align the proximal and distal fallopian tube segments prior to the reanastomotic step, 5-0 polyglactin 910 suture (Vicryl; Ethicon, San Angelo, TX, USA) was used in an anterior-to-posterior fashion at the distal aspect of the mesosalpinx and then in a posterior-to-anterior

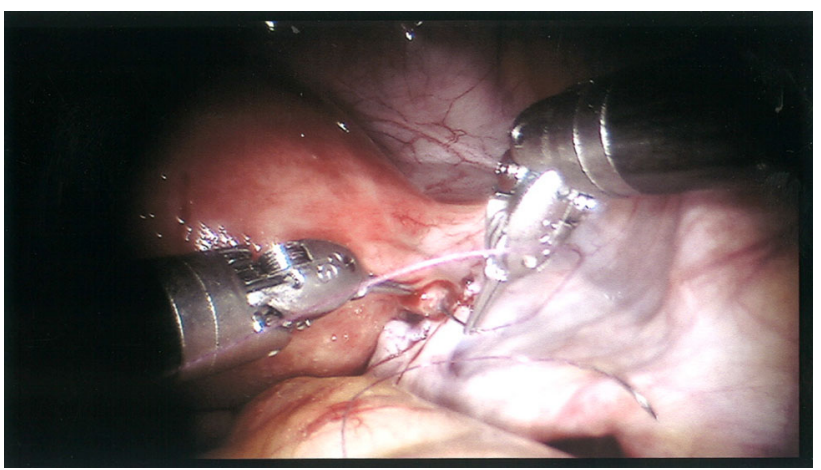

Fig. 1 Placement of 12 o'clock suture into the right fallopian tube's proximal lumen

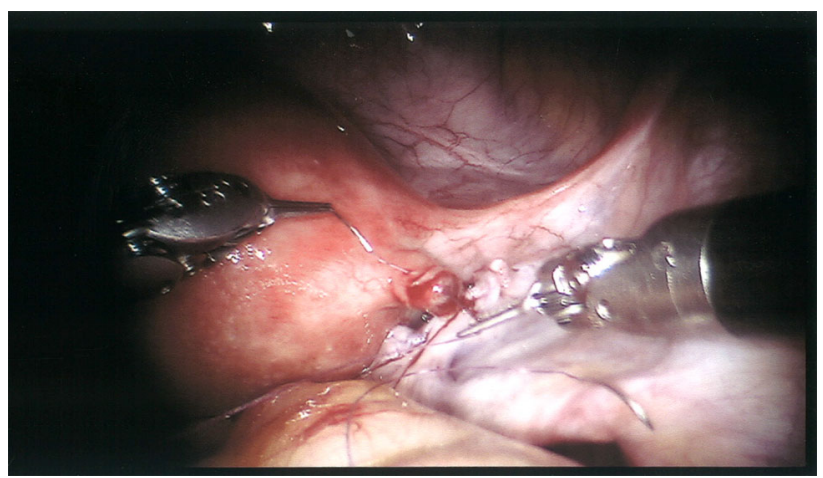

Fig. 2 Pulling the needle through the serosal surface of the right fallopian tube after 12 o'clock suture placement

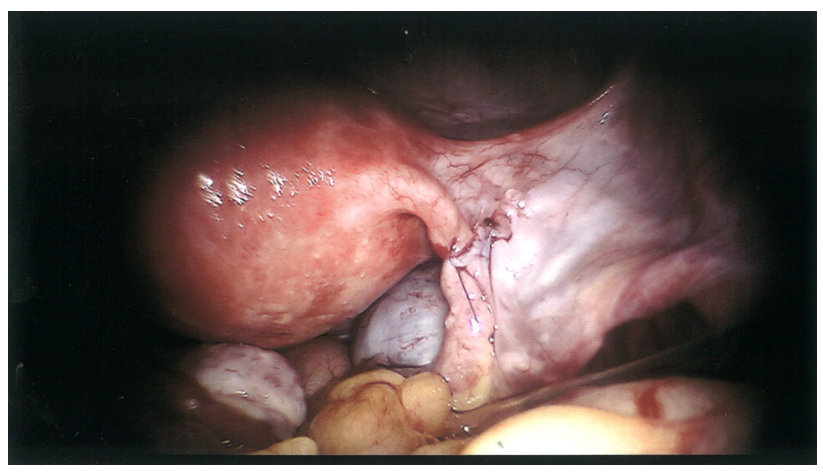

Fig. 3 Right fallopian tube after anastomosis

fashion at the proximal aspect of the mesosalpinx. The robotic instruments used as needle drivers for the mesosalpingeal stitch were two EndoWrist ${ }^{\circledR}$ Black Diamond Micro Forceps.

On both the proximal and distal segments' lumina, the one-stitch anastomotic step was performed by using a double-needle, $30 \mathrm{~cm}, 5-0$ polyglactin 910 suture into the lumen at the 12 o'clock position from the mucosa, into the muscularis, and out through the serosa (Figs. 1, 2). The robotic instruments used as needle drivers for the anastomotic step were two EndoWrist Black Diamond Micro Forceps. 


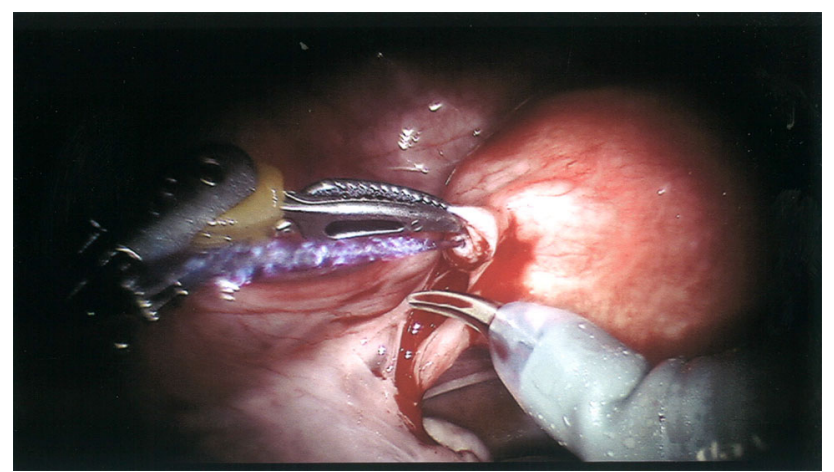

Fig. 4 Chromotubation of indigo carmine through the left fallopian tube's proximal segment prior to anastomosis

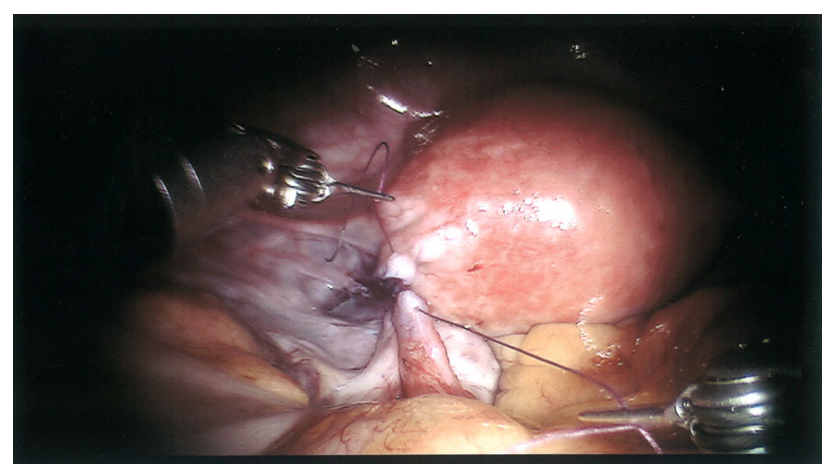

Fig. 5 Approximation of left fallopian tube's proximal and distal segments after 12 o'clock suture placement into each of the aforementioned segments

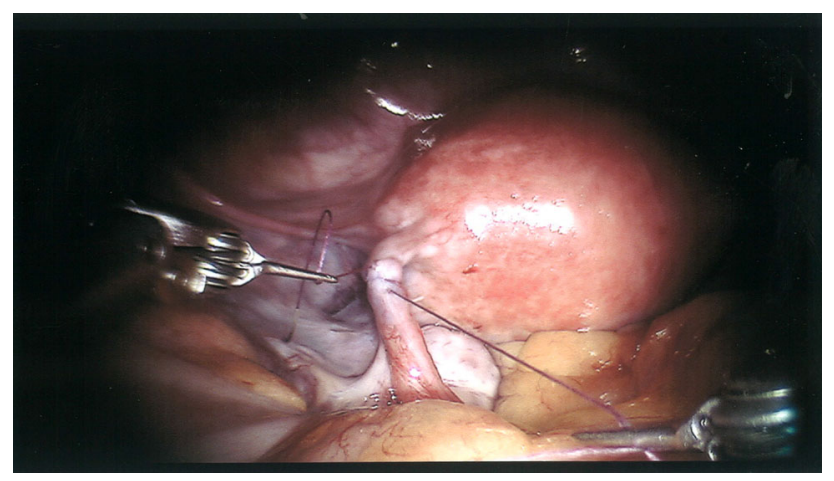

Fig. 6 Left fallopian tube after anastomosis

Chromotubation was performed after anastomosis and oxidized regenerated cellulose (Interceed, Ethicon Women's Health and Urology) was placed over and wrapped around each fallopian tube's anastomosis (Figs. 3, 4, 5, 6). All patients were discharged to home on the same day.

If a patient had not achieved pregnancy within 4-6 months after RATA, postoperative HSG was performed in order to assess tubal patency. For patients who had HSG performed, HSG reports were obtained and the films were reviewed as well.

\section{Results}

Eighteen patients underwent RATA (surgery performed by S.K.K.); one patient was lost to follow-up. The mean age of the 18 women was 33.7 years with a range of $27-39$. Blood loss was, on average, less than $25 \mathrm{ml}$ per surgical case. There were no intraoperative or postoperative complications with the surgical cases. The mean operative time for the 16 bilateral RATAs and one unilateral RATA with the one-stitch technique in this series was $146 \mathrm{~min}$ (range 89-203) as compared to 201 min (range 140-263) for 16 bilateral TAs and two unilateral TAs as reported by Dharia Patel [2].

In 16 of 17 patients (94.1\%), tubal patency was confirmed in at least one fallopian tube by postoperative HSG and/or subsequent pregnancy. Nine of the patients had postoperative HSG. Eight of these had bilateral TAs, of which five had bilateral tubal patency, two had unilateral patency, and one had bilateral blockage shown at HSG. One patient underwent unilateral TA and the HSG demonstrated unilateral tubal patency. The pregnancy rate, defined as a positive quantitative beta human chorionic gonadotropin level, was 10/17 (58.8\%). Seven patients had intrauterine pregnancies with fetal cardiac motion $(41.2 \%)$, one patient had an ectopic pregnancy (5.9\%), and two patients had spontaneous pregnancy losses $(11.8 \%)$, one of which was a blighted ovum and the other a chemical pregnancy. Of the ten women who achieved pregnancy, $8 / 10(80.0 \%)$ had sonographic evidence of intrauterine pregnancy (one of which was a blighted ovum), 1/10 (10\%) had an ectopic pregnancy, and 1/10 (10\%) had a chemical pregnancy. The average time from surgery to documented conception was 6 months (range 2-22).

\section{Conclusion}

RATA has been shown to be a feasible and cost-effective surgical enhancement; however, it has been shown to entail increased operative times when compared to the open approach [2, 6]. RATA with the one-stitch technique for the reanastomotic step appears to have similar patency rates as RATAs that are performed with multiple sutures placed for the reanastomotic step [2]. Since the tubal anastomotic step in RATA typically is performed with multiple interrupted sutures (usually four), the one-stitch anastomotic step may decrease operative times while achieving comparable patency and pregnancy rates [2]; in addition, the lower number of sutures placed may theoretically decrease the chances of restenosis of the fallopian tube [10]. Further studies with head-to-head comparison would be needed to compare the operative times between the multi-stitch and single-stitch methods. 
Acknowledgments We acknowledge Austin Fertility and Reproductive Medicine/Westlake IVF (Kate Odenwald, R.N., A.C.N.P.B.C.), surgical staff at St. David's South Austin Medical Center in Austin, Texas (Charlie Harlan, C.S.T., Tara Buck, R.N., B.S.N., C.N.O.R., Thomas Butz, R.N.), and surgical staff at the Hospital at Westlake Medical in Austin, Texas (Robert Currie, Jr.).

Conflict of interest Author Shahryar K. Kavoussi declares that he has no conflict of interest. Author K. M. Kavoussi declares that he has no conflict of interest. Author Dan I. Lebovic has received research grants from AbbVie Pharmaceutical, Inc.

Informed consent No animals were used in this study. Informed consent was not obtained due to the retrospective (chart review) nature of the study; IRB approval was obtained.

\section{References}

1. Hirshfeld-Cytron J, Winter J (2008) Laparoscopic tubal reanastomosis versus in vitro fertilization: cost-based decision analysis. Am J Obstet Gynecol 209:56.e1-56.e6

2. Dharia Patel SP, Steinkampf MP, Whitten SJ, Malizia BA (2008) Robotic tubal anastomosis: surgical technique and cost effectiveness. Fertil Steril 90(4):1175-1179
3. Degueldre M, Vandromme J, Huong PT, Cadière GB (2000) Robotically assisted laparoscopic microsurgical tubal reanastomosis: a feasibility study. Fertil Steril 74(5):1020-1023

4. Goldberg JM, Falcone T (2003) Laparoscopic microsurgical tubal anastomosis with and without robotic assistance. Hum Reprod 18(1):145-147

5. Vlahos NF, Bankowski BJ, King JA, Shiller DA (2007) Laparoscopic tubal reanastomosis using robotics: experience from a teaching institution. J Laparoendosc Adv Surg Tech A 17(2): $180-185$

6. Rodgers AK, Goldberg JM, Hammel JP, Falcone T (2007) Tubal anastomosis by robotic compared with outpatient minilaparotomy. Obstet Gynecol 109(6):1375-1380

7. Caillet M, Vandromme J, Rozenberg S, Paesmans M, Germay O, Degueldre M (2010) Robotically assisted laparoscopic microsurgical tubal reanastomosis: a retrospective study. Fertil Steril 94(5): 1844-1847

8. Dubuisson JB, Swolin K (1995) Laparoscopic tubal anastomosis (the one stitch technique): preliminary results. Hum Reprod 10(8):2044-2046

9. Bissonnette F, Lapensée L, Bouzayen R (1999) Outpatient laparoscopic tubal anastomosis and subsequent fertility. Fertil Steril 72(3):549-552

10. Tusheva OA, Gargiulo AR, Einarsson JI (2013) Application of robotics in adnexal surgery. Rev Obstet Gynecol 6(1):e28-e34 